\title{
AODV with intelligent priority flow scheme for multi-hop ad hoc networks
}

\author{
Zne-Jung Lee ${ }^{1} \cdot$ So-Tsung Chou ${ }^{2} \cdot$ Chou-Yuan Lee ${ }^{3} \cdot$ Bin-Yu Peng $^{2}$
}

Received: 10 March 2016 / Accepted: 2 June 2016 / Published online: 17 June 2016

(c) The Author(s) 2016. This article is published with open access at Springerlink.com

\begin{abstract}
A mobile ad hoc network is formed by a cluster of mobile stations randomly located within a certain area without the infrastructure of base stations. There have been many researches proposing different models of routing protocols and numerous simulations to enhance their performances under varying conditions and constraints. However, most of them only focus on network layer without considering the influence of media access control (MAC) layer. In this paper, a new strategy of cross layer algorithm is proposed based on the characteristics of reactive routing and multi-hop jumping. In addition, artificial bee colony algorithm is performed to calculate the contention window of the nodes along the routing path over MAC layer. This framework could avoid data collision in MAC layer, and results in getting better performances of data transmission in network layer. Extensive simulations show that the proposed AODV with intelligent priority flow scheme is able to earn a significant improvement over the conventional routing algorithm.
\end{abstract}

Keywords AODV protocol C Contention window · Artificial bee colony algorithm (ABCA) .

Mobile ad hoc network (MANET)

Zne-Jung Lee

johnlee@cc.hfu.edu.tw

1 Department of Information Management, Huafan University, New Taipei City, Taiwan

2 Department of Information Management, University of Kang Ning, Tainan City, Taiwan

3 Department of Information Management, Lan Yang Institute of Technology, Yilan, Taiwan

\section{Introduction}

With the advancement of wireless technology, people have witnessed an ever-increasing popularity of wireless networks in recent years. Wireless local area networks, or Wi-Fi hotspots, have been widely deployed in cities, college campus, airports, coffee bars, conference halls, hotels, and many other public places. Nevertheless, wireless local area network is limited to one-hop communication between clients and access points. It restricts wireless access to a small range. If communication devices are allowed to forward packets for others, a multi-hop ad hoc network can be formed, and the range of wireless access to Wi-Fi hotspots can be significantly extended. This kind of wireless multi-hop communication could be used in many applications, such as environmental monitoring and health care.

In multi-hop ad hoc network, nodes communicate with each other using wireless links of each node. Each node acts as a host as well as a router and forwards data packets for other nodes. A central challenge in the design of multihop ad hoc networks is the development of dynamic routing protocol that can efficiently find routes between two communication nodes. Many protocols, such as dynamic destination sequenced distance vector (DSDV) [1], dynamic source routing (DSR) [2], ad hoc on-demand distance vector (AODV) [3], temporally ordered routing algorithm (TORA) [4], and zone routing protocol (ZRP) [5], etc, have been proposed. However, we focus on another challenge in the efficiency of data transmission for multi-hop ad hoc networks. Wireless media is a shared and scarce resource in an ad hoc network. How to efficiently control the access to this shared media becomes an important and complicated issue. For multi-hop ad hoc networks, nodes have to cooperate to forward each other's packets through the network. Due to the contention for the shared channel, the throughput of each single node 
is limited not only by channel capacity itself but also by the transmissions in neighborhood. That is to say, the transmission at each hop has to contend the channel with upstream and downstream nodes. This effect results in congestion at some nodes along the routing path and seriously limits the performances of multi-hop ad hoc networks. Li et al. [6] have found that the IEEE 802.11 mechanisms fail to achieve the optimal scheduling for all multi-hop flows, and its performance greatly degrades under the chain topology with heavy load.

To alleviate the congestion, several dynamic load balancing algorithms were proposed. Lee and Gerla presented a dynamic load-aware routing algorithm (DLAR) [7] which uses the traffic load of the intermediate nodes as route selection criterion. It periodically monitors the status of active data sessions and dynamically reconfigures the routes that are being congested. Lee and Campbell presented a hotspot mitigation protocol (HMP) [8], where hotspots represent transient but highly congested regions. HMP balances resource consumption among neighboring nodes by suppressing new route requests and controlling TCP flows rate. These solutions focus only on routing algorithms and do not fully consider the MAC layer contentions which result in different problems of channel access at the neighboring nodes. Recently, intelligent algorithms, such as genetic algorithm (GA), ant colony optimization (ACO), and particle swarm optimization (PSO), have shown good performances in various problems [9-13]. A flexible genetic algorithm for optimizing channel assignment in mesh wireless networks was proposed by Vanhatupa et al. [10]. In Alba [11], a specialized cellular multi-objective genetic algorithm was used for finding an optimal broadcasting strategy in urban mobile ad hoc network (MANET). ACO has been successfully adapted for implementing new routing protocols for MANET [12]. Huang et al. [13] proposed a new routing protocol based on a PSO to make scheduling decisions for reducing the packet loss rate. In this paper, AODV with intelligent priority flow scheme for multi-hop ad hoc networks is proposed. In the proposed approach, ABCA will be used to calculate the contention window size of the nodes along the routing path. It can avoid data collision in MAC layer, and can result in getting better performances of data transmission.

The remaining part of this paper is organized as follows. In Sect. 2, we briefly review AODV protocol, IEEE 802.11 DCF standard, and ABCA algorithm. Section 3 introduces the proposed AODV with intelligent priority flow scheme. The simulation results are presented in Sect. 4. Finally, we conclude this paper in Sect. 5.

\section{Background and related work}

In this section, an overview of two related protocols and ACBA algorithm is given. As our target network is a multi- hop ad hoc network, AODV routing protocol is adopted to find routing path in the network layer, IEEE 802.11 distributed coordination function (DCF) is used as the medium-access control protocol in the MAC layer, and ACBA algorithm uses to calculate the contention window size of the nodes along the routing path.

\subsection{Overview of AODV routing protocol}

AODV is an on-demand dynamic routing protocol that uses routing tables with one entry per destination. When a source node needs a route to a destination, it initiates a route discovery process to locate the destination node. The source node floods a query packet, i.e., route request (RREQ), requesting a route to be set up to the destination. A reply, i.e., route reply (RREP), is sent back directly to the source node either by the destination itself or any other intermediate node that has a current route to the destination. On receiving a route request, intermediate nodes update their routing table for the reverse route to the source. Similarly, the forward route to the destination is updated on receiving a route reply packet. AODV uses sequence numbers to determine the timeliness of each packet and to prevent loops. Expire timers are used to keep the route entries fresh.

Link failures are propagated by a route error (RERR) message from the site of a link break to the source node for that route. When the next hop link breaks, RERR packets are sent to a set of neighboring nodes that communicate over the broken link with the destination. This recursive process erases all broken entries in the routing table of the nodes. Since nodes reply to the first arriving RREQ, AODV favors the least congested route instead of the shortest route. The AODV on-demand approach minimizes routing table information. However, this potentially leads to a large number of route requests being generated [14].

\subsection{Overview of IEEE 802.11 DCF standard}

This subsection briefly summarizes the distributed coordination function (DCF) as standardized by the IEEE 802.11 Working Group. For a more complete and detailed presentation, please refer to the IEEE 802.11 standard [15]. A station with a new packet for transmission needs to monitor the channel activity first. If the channel is idle for a period of time equal to the distributed inter-frame space (DIFS), the station starts to transmit instantly. Otherwise, the channel is busy and the station persists to monitor the channel until it is measured idle for a DIFS. At this point, the station generates a random back-off interval before transmitting to minimize the probability of multiple stations simultaneously starting transmission. Furthermore, to avoid channel capture, a station must wait a random back-off time between two consecutive packet transmissions, even if the medium is sensed idle for a 


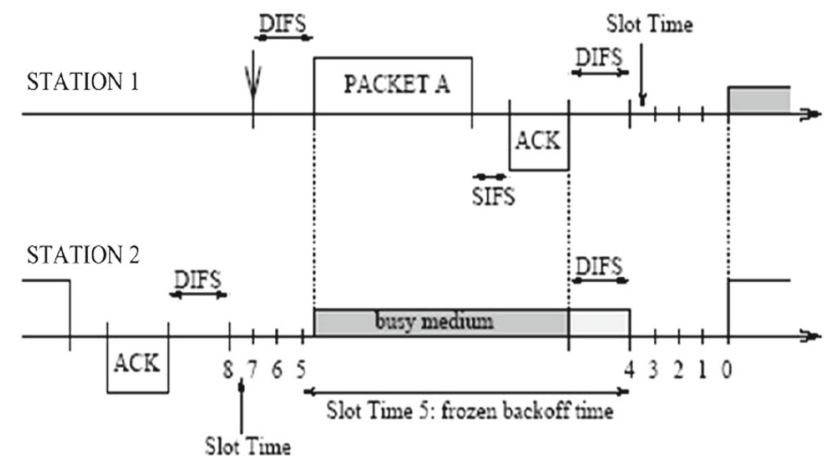

Fig. 1 Illustration of basic access and back-off mechanism of DCF

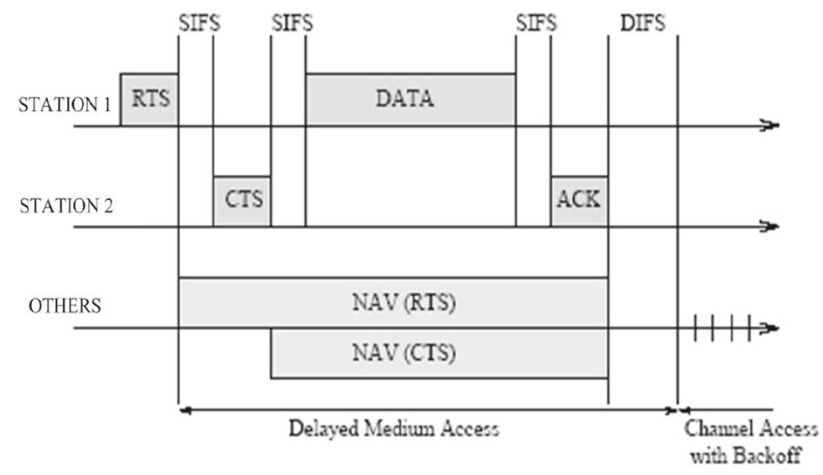

Fig. 2 Illustration of RTS/CTS and back-off mechanism of DCF

DIFS time period after the previous transmission. An ACK is transmitted by the destination to signal the source about the successful packet reception after a short inter-frame space (SIFS) at the end of the received packet.

The two-way handshaking technique for packet transmission described above is called basic access mechanism, as shown in Fig. 1. DCF also defines an optional four-way handshaking technique for packet transmission. This mechanism, also known as RTS/CTS, is shown in Fig. 2. A station that has a packet queued for transmission follows the back-off rules explained above, but instead of transmitting the packet it preliminarily transmits a special short frame called requestto-send (RTS). When the destination detects an RTS frame, it responds after an SIFS time period with a clear to send (CTS) frame. The source is only allowed to transmit the data packet if the CTS frame is correctly received within a duration called CTS_ Timeout. The RTS threshold (RT) is a switch parameter for the RTS/CTS mechanism; four-way handshaking is used when the packet for transfer is larger than the RT value, otherwise two-way handshaking is used.

\subsection{Overview of artificial bee colony algorithm (ABCA)}

A recently introduced optimization algorithm, artificial bee colony algorithm (ABCA), is proposed by Karaboga [16]. $\mathrm{ABCA}$ simulates the foraging behavior of a bee colony into three groups of bees: employed bees, onlooker bees, and scout bees. In ABCA, half of the artificial bee colony consists of employed bees and the other half constitutes onlooker bees. Employed bees investigate the food source and share their food information to onlooker bees. The higher quality of food source, the larger probability will be selected by onlooker bees. The employed bee becomes a scout bee when the employed bee abandons its food source and search for new one. The details of ABCA are described as follows.

ABCA starts with randomly producing the initial food sources that correspond to the solutions for employed bees. The initial population of solutions is filled with $S N$ number of randomly generated $D$-dimensional food sources

$F\left(\boldsymbol{X}_{i}\right), \boldsymbol{X}_{i} \in R^{D}, \quad i \in\{1,2,3, \ldots, S N\}$

where $\boldsymbol{X}_{i}=\left[x_{i 1}, x_{i 2}, \ldots, x_{i D}\right]$ is the position of the $i^{t h}$ food source and $F\left(\boldsymbol{X}_{i}\right)$ is the cost function which represents the quality of the $i$ th food source. To update a feasible food source (solution) position $\boldsymbol{V}_{i}=\left[v_{i 1}, v_{i 2}, \ldots, v_{i D}\right]$ from the old one $\boldsymbol{X}_{i}$, the ABCA uses Eq. (2) as follows:

$v_{i j}=x_{i j}+\varphi_{i j}\left(x_{i j}-x_{k j}\right)$.

In Eq. (2), $v_{i j}$ is a new feasible solution, $k \in\{1,2,3, \ldots, S N\}$ and $j \in\{1,2,3, \ldots, D\}$ are randomly chosen indexes, $k$ has to be different from $j$ and $\varphi_{i j}$ is a random number in the range $[-1,1]$. After all employed bees complete their searches, they share their information related to the nectar amounts and food sources positions with the onlooker bees on the dance area. An onlooker bee evaluates the nectar information taken from all employed bees. In addition, the probability for an onlooker bee chooses a food source can be obtained from below.

$P_{i}=\frac{\mathrm{fit}_{i}}{\sum_{n=1}^{\mathrm{SN}} \mathrm{fit}_{n}}$

where fit ${ }_{i}$ is the fitness value of the $i$ th food source related to the cost function $F(\boldsymbol{X} i)$. If a food source cannot be further improved through a predetermined number of iterations, the food source is assumed to be abandoned, and the corresponding employed bee becomes a scout bee. The new random position chosen by the scout bee is described as follow.

$x_{i j}=x_{j}^{\min }+\operatorname{rand}[0,1] *\left(x_{j}^{\max }-x_{j}^{\min }\right)$

where $x_{j}^{\min }$ is the low bound and $x_{j}^{\max }$ is the upper bound of the food source position in dimension $j$. 


\section{AODV with intelligent priority flow scheme}

In this paper, AODV with the intelligent priority flow scheme for multi-hop ad hoc networks is proposed. In the proposed approach, all the routing algorithms (e.g., route discovering and path maintaining) are set as the same as original AODV protocol in network layer. In the IEEE 802.11 DCF standard, the contention window $(\mathrm{CW})$ is an important variable to determine back-off time of each node. Thereafter, ABCA is used to calculate the $\mathrm{CW}$ size for each node along the routing paths. Then, the $\mathrm{CW}$ size is descending from source node to destination node. This strategy of the proposed approach could achieve optimal packet scheduling, avoid data collision in MAC layer, and result in getting better performances of data transmission in network layer. In AODV with the intelligent priority flow scheme, the $\mathrm{CW}$ and back-off time are performed as follows:

$\mathrm{CWMax}=\alpha \quad(32 \leq \alpha \leq 1024)$

CWMin $=1024 /\left(\boldsymbol{\beta}^{\mathbf{L}-\mathbf{D}}\right) \quad(1 \leq \boldsymbol{\beta} \leq 10)$

$L$ total hop count of routing path, $D$ the remain hop count of AODV routing table,

CW CWMin $* \gamma^{n-1}$ slots $(1 \leq \gamma \leq 10)$,

$n$ the number of data retransmitted times.

$\mathrm{CWMin} \leq \mathrm{CW} \leq \mathrm{CWMax}$

If (transmit successfully), then reset CW $=$ CWMin Descending CW from source node to destination node

Back-off time $=$ int $[\mathrm{CW} * \operatorname{random}()] *$ slot time random(): a random real number between 0 and 1 int [expression]: the lowest integer for expression

where $\alpha$ is the upper bound integer value determined by $\mathrm{ABCA} ; \boldsymbol{\beta}$ is the lower bound integer $(1 \leq \boldsymbol{\beta} \leq 10)$ for CWMin determined by $\mathrm{ABCA} ; \gamma$ is a descending integer $(1 \leq \gamma \leq 10)$ for $\mathrm{CW}$ determined by $\mathrm{ABCA}$.

Thereafter, $\mathrm{ABCA}$ is performed to find the best values for above three parameters $(\alpha, \boldsymbol{\beta}, \gamma)$. First, the initial population and solutions for all employed bees are generated in ABCA. The employed bee goes to a solution and determines a neighbor source, as shown in Eq. (2), then evaluates its quality of solution. Each onlooker watches the dance of employed bees and chooses one of their sources, and then goes to that source, as shown in Eq. (3). Abandoned food sources are determined and are replaced with the new food sources discovered by scouts, as shown in Eq. (4). The maximum number of iterations (MNI) is used to control the number of iterations as the termination criterion. ABCA will be repeated until the output of the value of fitness reaches the pre-defined threshold value or the number of iterations meets MNI. Two metrics of performance are evaluated to illustrate as the value of fitness. One is packet delivery ratio, and the other is end-to-end throughput. Packet delivery ratio (PDR) is defined as the percentage of data packets that can received by the destination. End-to-end throughput (ETEH) is defined as total amount of data that can be received by the destination in a given time interval. Frame cost of destination per data (FCDPD) is defined as the ratio of total frames, including RTS, CTS, ACK, RREQ, RERR, RREP, and CBR data, delivered by the source, to the number of CBR data received successfully by destination. RREQ number of route discovery (RREQN) is defined as how many number of RREQ packets were sent by the source node during data transmission. RERR number of route maintenance (RERRN) is defined as how many number of RERR packets were received by the source node during data transmission. In the proposed AODV with the intelligent priority flow scheme, the value of fitness is set as follows:

$$
\begin{aligned}
\text { fiti }= & \text { PDRi }+ \text { ETEHi }+1 / \text { FCDPDi } * 100 \\
& +1 / \text { RREQNi } * 100+1 / \text { RWEENi } * 100 .
\end{aligned}
$$

\section{Simulation results}

The simulation was implemented under the network simulator NS2 2.29, which can simulate a layered network protocol stack and wireless channel [17]. The simulation scenario is conducted in a 7-nodes chain topology of multi-hop ad hoc networks, as shown in Fig. 3. The distance between neighboring nodes is $200 \mathrm{~m}$, which allows a node to connect only to its neighboring nodes. The same distance between neighboring nodes ensures that the nodes act equally in the simulation. The number of employed bees and onlooker bees were set to 10 . The MNI was set to 2000. The IEEE 802.11 DCF is used as the medium-access control protocol. All nodes communicate with identical, half duplex, and wireless radios that have a bandwidth of $1 \mathrm{Mbps}$, an effective transmission radius of $250 \mathrm{~m}$ and the interfering range of $500 \mathrm{~m}$. Each node has a queue called interface queue (IFQ) for packets waiting to be transmitted by the network interface, which holds up to

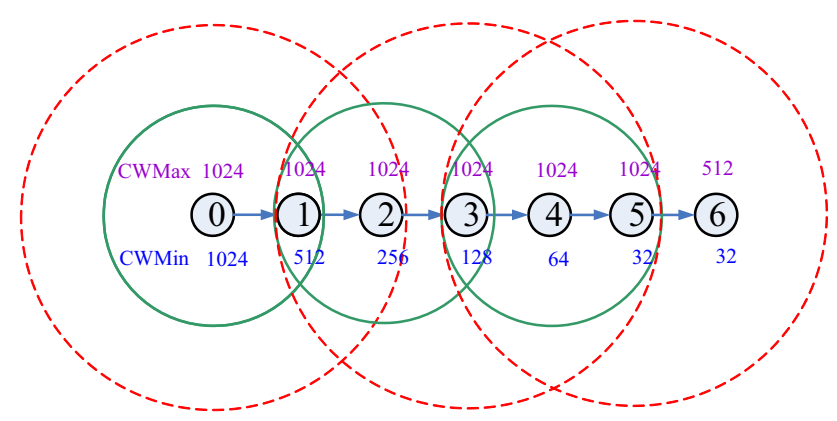

Fig. 3 A 7-nodes chain topology for multi-hop ad hoc network 


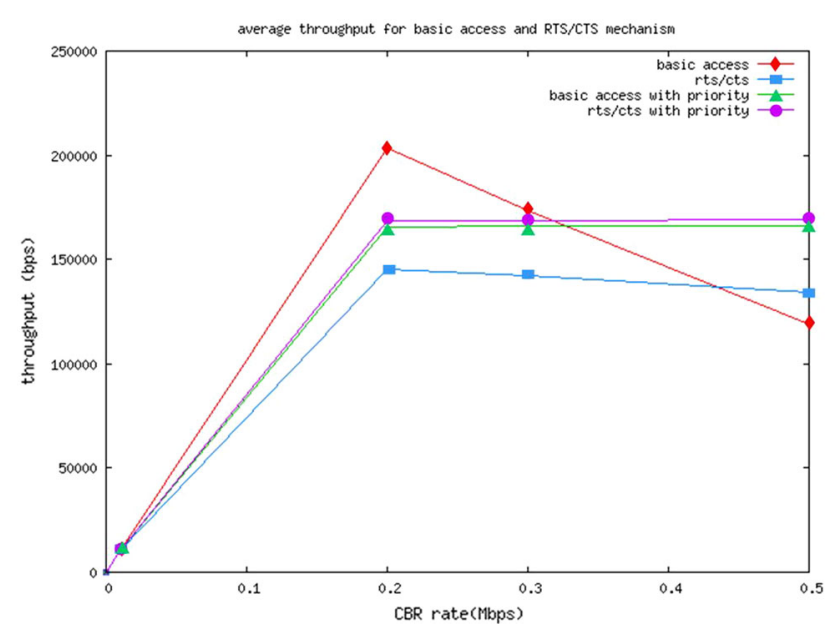

Fig. 4 End-to-end throughput in the 7-nodes chain topology

10 packets and is managed in a drop tail fashion. The tworay ground reflection model is used for propagation. AODV routing protocol is adopted to find routing path in network layer. In this paper, constant bit rate (CBR) traffic is used to simulate the UDP performance. Every CBR packet size is 1200 bytes, and the packet sending rate is varied in each run to change the offered traffic load $(0.01,0.2,0.3$, and 0.5 Mbps) in the network. To demonstrate the performance of the proposed approach in a fair comparison, ABCA is performed to find the best values for three parameters $(\alpha, \beta, \gamma)$ first. Thereafter, all compared simulations take $100 \mathrm{~s}$. Simulation will evaluate and compare the performances between the original AODV base on the IEEE 802.11 DCF and our proposed scheme (AODV-SPF). In the following figures, our scheme will be referred to as basic access with priority and RTS/CTS with priority relative to the traditional mechanisms of the IEEE 802.11.

\section{A. End-to-end throughput}

Figure 4 shows that our scheme improves the end-to-end throughput of AODV for both basic access and RTS/CTS mechanisms under heavy traffic load. The throughput of CBR packet based on basic access is little higher than that for priority flow mechanism under light traffic load, but the delivery ratio is less than that for priority flow, as shown in Fig. 5. It means that the traditional basic access mechanism sends more CBR packets than our scheme, and some of them are lost. The throughput of CBR packet with priority flow is much higher and more stable than that for RTS/CTS mechanism over all traffic loads, and the delivery ratio is obviously larger than that for RTS/CTS, as shown in Fig. 6.

\section{B. Packet delivery ratio}

Figure 5 shows that the AODV-SPF has much higher packet delivery ratio than original AODV for both basic and RTS/CTS mechanisms independent of traffic load. The

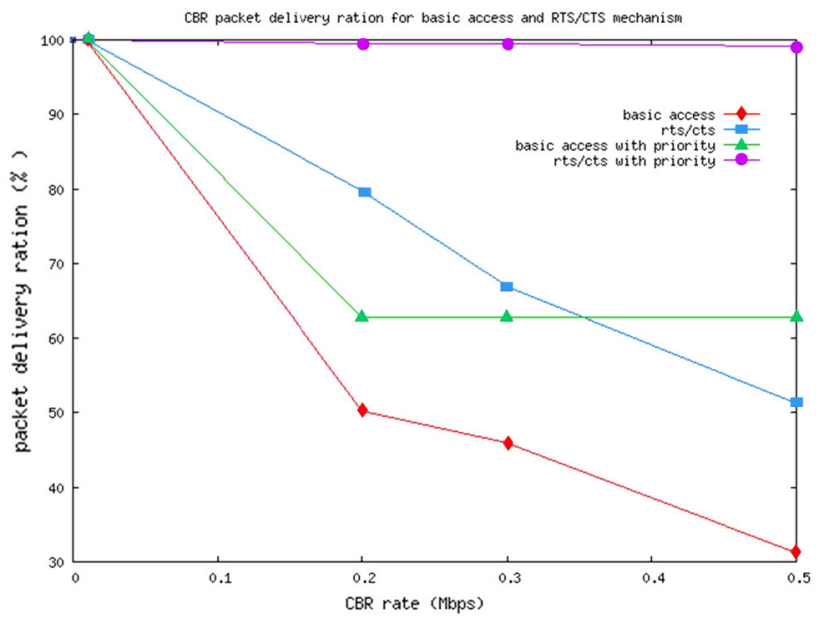

Fig. 5 Packet delivery ration in the 7-nodes chain topology

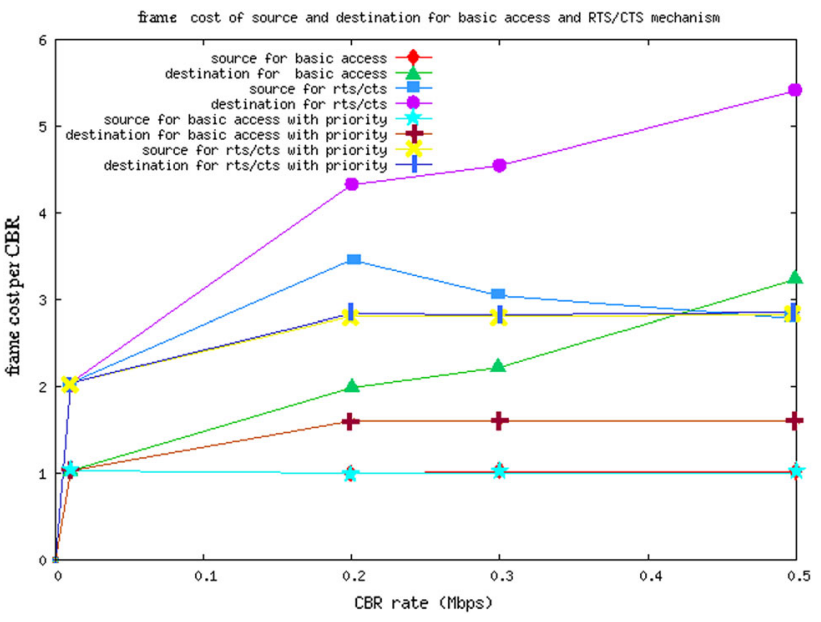

Fig. 6 Normalized control overhead in the 7-nodes chain topology

packet delivery ratio of CBR data for our scheme is much higher than that for basic access mechanism all the time without increasing the overhead cost of CBR data, as shown in Fig. 6. Moreover, it is almost approach to 100 transmission for RTS/CTS. That is to say, both kinds of priority flow mechanisms may suffer less collision than the traditional mechanisms the whole time while transmitting CBR packet.

\section{Normalized control overhead}

Figure 6 shows that AODV-SPF keeps normalized control overhead small and stable, and has the difference of frame cost between source and destination much smaller than that of original AODV. This verifies that our scheme reduces a lot of collisions in MAC layer, and saves unsuccessful RTS/CTS negotiations or RREQ, RREP, and REPP route control packets. The original AODV has a much higher normalized control overhead, which increases rapidly with the offered load for multi-hop flow. This implies that the AODV- 


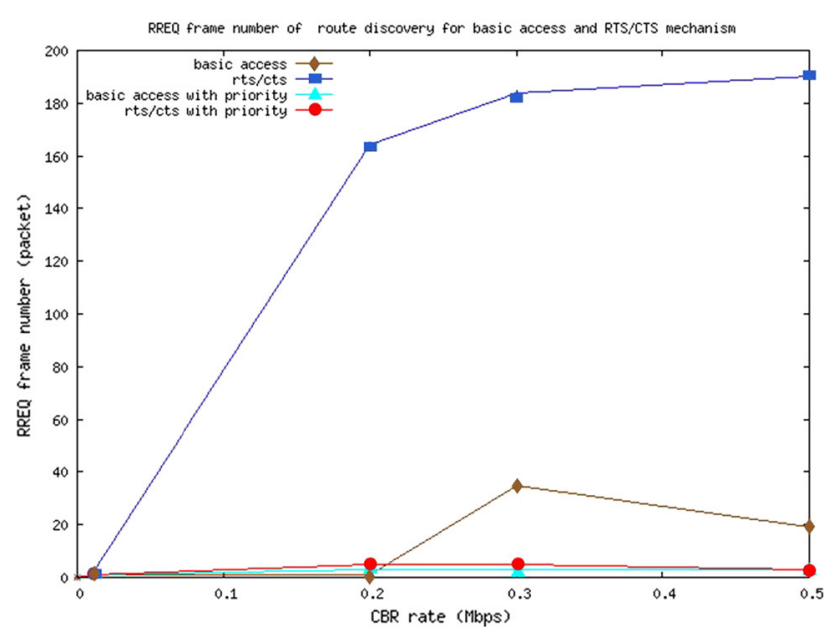

Fig. 7 RREQ packet number of route discovery in the 7-nodes chain topology

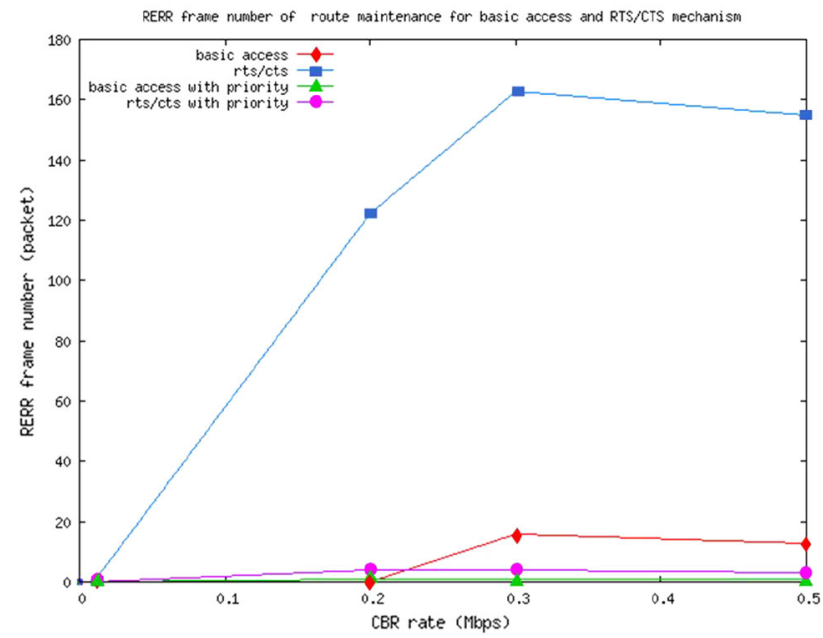

Fig. 8 RERR packet number of route maintenance in the 7-nodes chain topology

SPF is a better choice than the traditional schemes for the multi-hop flow in the shared wireless channel environment and is scalable for larger network.

\section{Probability of link failure}

Figures 7 and 8 show that the AODV-SPF has absolutely smaller number of route control packets, e.g., RREQ and RERR, than original AODV based on the IEEE 802.11. In Fig. 8, the maximum number of RREQ sent by source node is about 5 packets in AODV-SPF, but 190 packets in original AODV based on RTS/CTS, and about 35 packets based on basic access mechanism. Relatively, as shown in Fig. 8, the maximum number of RERR received by source node is about 4 packets in our scheme, but 163 packets in original AODV based on RTS/CTS, and about 16 packets based on basic access mechanism. Based on the above description, we prove that our scheme is superior to the original AODV.

\section{Conclusions}

In this paper, we discuss the intra-flow contention which causes poor performance of the IEEE 802.11 DCF MAC protocol in multi-hop ad hoc network. To alleviate this contention, we propose a framework of media access scheduling algorithm based on the hop count number that packets have traversed. In the proposed approach, these best values of parameters are performed by ABCA. Simulation results verify that the proposed AODV with the intelligent priority flow scheme could achieve obviously better performance of data transmission, such as more stable and higher throughput, better packet delivery ratio, lower routing load, and decreasing the control packet number of RREQ and RERR than the original AODV. In view of the above descriptions, it is shown that the proposed approach is superior to original AODV.

Open Access This article is distributed under the terms of the Creative Commons Attribution 4.0 International License (http://creativecomm ons.org/licenses/by/4.0/), which permits unrestricted use, distribution, and reproduction in any medium, provided you give appropriate credit to the original author(s) and the source, provide a link to the Creative Commons license, and indicate if changes were made.

\section{References}

1. Amirhosein, I., Alireza, K.H.: DSDV-Het; a new scalable routing protocol for large heterogeneous ad Hoc networks. In: Telecommunications (IST), 7th International Symposium on, pp. 590-595 (2014)

2. Long, Z., He, Z.: Optimization and implementation of DSR route protocol based on ad hoc network. In: International Conference on Wireless Communications, Networking and Mobile Computing, pp. 1508-1511 (2007)

3. Yang, H., Li, Z.Y.: Simulation and analysis of a modified AODV routing protocols. In: Computer Science and Network Technology (ICCSNT), International Conference on, pp. 1440-1444 (2011)

4. Rajeswari, M., Maheswari, P.U., Bhuvaneshwari, S., Gowri, S.: Performance analysis of AODV, DSR, TORA and OLSR to achieve group communication in MANET. In: 4th International Conference on Advanced Computing (ICoAC), pp. 1-8 (2012)

5. Loutfi, A., Elkoutbi, M.: Evaluation and enhancement of ZRP performances. In: Multimedia Computing and Systems (ICMCS), International Conference on, pp. 1-6 (2011)

6. Li, J., Blake, C., Couto, D., Lee, H., Morris, R.: Capacity of ad hoc wireless network. In: Proceedings of ACM MobiCom, 2001

7. Lee, S.J., Gerla, M.: Dynamic load aware routing in ad hoc networks. In: Proceedings of IEEE ICC, 2001

8. Lee, S.B., Campbell, A.T.: HMP; hotspot mitigation protocol for mobile ad hoc networks. In: 11th IEEE/IFIP International Workshop on Quality of Service (IWQOS 2003)

9. Jose, G.N., Enrique, A.: Automatic parameter tuning with metaheuristics of the AODV routing protocol for vehicular ad hoc networks. In: Di Chio, C., Brabazon, A., Di Caro, G.A., Ebner, M., Farooq, M., Fink, A., Grahl, J., Greenfield, G., Machado, P., O’Neill, M., Tarantino, E., Urquhart, N, (eds.) Applications of Evolutionary Computation, EvoApplications 2010: EvoCOMNET, EvoENVIRONMENT, EvoFIN, EvoMUSART, and EvoTRANSLOG, Istanbul, Turkey, April 7-9, 2010, Proceedings, Part 
II. Lecture Notes in Computer Science, pp. 21-30. Springer, Berlin, Heidelberg (2010)

10. Vanhatupa, T., Hannikainen, M., Hamalainen, T.: Optimization of mesh WLAN channel assignment with a configurable genetic algorithm. In: WiMeshNets, 2006

11. Alba, E.: A cellular MOGA for optimal broadcasting strategy in metropolitan MANETs. Comput. Commun. 30(4), 685-697 (2007)

12. Di-Caro, G.A., Ducatelle, F., Gambardella, L.M.: AntHocNet: an adaptive nature-inspired algorithm for routing in mobile ad hoc networks. Eur. Trans. Telecommun. 16(5), 443-455 (2005)

13. Huang, C., Chuang, Y., Hu, K.: Using particle swarm optimization for QoS in ad hoc multicast. Eng. Appl. Artif. Intell. 22, 1188-1193 (2009)

14. Singh, M., Sharma, J.: Performance analysis of secure and efficient AODV (SE-AODV) with AODV routing protocol using NS2. Reliability, In: Infocom Technologies and Optimization (ICRITO) (Trends and Future Directions), 3rd International Conference on, pp. 1-6 (2014)
15. IEEE 802.11 Working Group Part 11: Wireless LAN medium access control (MAC) and physical layer (PHY) specifications. ANSI/IEEE Std. 802.11 (1999)

16. Karaboga, D.: An idea based on honey bee swarm for numerical optimization. Technical Report-TR06, Erciyes University, Engineering Faculty, Computer Engineering Department, Turkey (2005)

17. Fall, K., Varadhan, K.: Ns notes and documentation. LBNL (1998). http://www.mash.cs.berkeley.edu/ns/. Accessed 26 June 2015 